ISSN 1112-9867

Available online at

http://www.jfas.info

\title{
DESIGN OF GRAPHIC AND ANIMATION IN GAME INTERFACE BASED ON \\ CULTURAL VALUE: VERIFICATION
}

\author{
R. Z. Ramli ${ }^{1, *}$, N. A. M. Zin ${ }^{2}$, N. Sahari@Ashaari ${ }^{2}$, M. N. Ismail ${ }^{3}$ and S. Osman ${ }^{4}$ \\ ${ }^{1}$ Faculty of Computer and Mathematical Sciences, Universiti Teknologi MARA, 72000 Kuala \\ Pilah, Negeri Sembilan, Malaysia \\ ${ }^{2}$ School of Information Technology, Faculty of Information Science and Technology, \\ Universiti Kebangsaan Malaysia, Bangi, Selangor, Malaysia \\ ${ }^{3}$ Faculty of Information Management, Universiti Teknologi MARA, Machang 18500, \\ Kelantan, Malaysia \\ ${ }^{4}$ Faculty of Industrial Information Technology, Universiti Selangor, 45600 Bestari Jaya, \\ Selangor, Malaysia
}

Published online: 17 October 2017

\begin{abstract}
A good interface design influences games players to continuously playing the game naturally. Different game players may have certain preferences based on their cultural background. It is important for the game designer to understand their target player. The goal of the research is to identify player preferences on game interface specifically on graphic and animation design based on their cultural factor. The four cultural values are context, PDI, IDV and MAS. This research applies triangulation method; questionnaire, experts'verification, interview. Analysis of the survey and the game interface design is done to get the outcome. The heat map generated shows nearly all of the seven respondents looked at the graphics and animations on the game interface. Interviews is then conducted where the outcome shows the gamers think the added localized graphics and animations give excitement and add presence to the game.
\end{abstract}

Keywords: game interface; cultural value; hofstede; prototype; eye tracker.

Author Correspondence, e-mail: ratna@ns.uitm.edu.my

doi: http://dx.doi.org/10.4314/jfas.v9i5s.14 


\section{INTRODUCTION}

Computer game is defined as a digital game with objective that brings enjoyment to the player

[1]. It has certain rules that limit the game playing. By using control devices such as keyboard, mouse or joys tick, player can interact with the game through the interface and receive visual or audio feedbacks. Good interface designs will inadvertently push the players to continuously playing by letting them feel that they have control over the game naturally [2]. There are several factors that influence the players to feel as if the interface design is acceptable and familiar to them. On top, cultural values are identified as a factor that control how a person acts and believes [3]. Product design such as game usually reflects its designer's culture [4]. Since current game industry is monopolized by American and Japanese, thus the designs are influenced by their respective culture. However, different players may have certain preferences based on their cultural background [5]. Thus, the game designer must understand their target player in order to design game. Past research [1] argues that the design of a good game interface should involve the target players during the development process. Evaluation by players must be done as early and as often as possible. Because culture influenced their behavior and online interaction, many researchers wondered about the importance of culture in virtual interaction [6-7]. Yet, information on the influence of culture on the motivation and behavior of users is limited $[4,8]$. Therefore, the goal of the research is to identify player preferences on game interface specifically on graphic and animation design based on their cultural factor.

Based on past researches context, PDI and MAS have been found to influence the design of graphic while context, MAS and IDV influenced the design of animation [5]. There are two research questions generated:

Q1: Do context, PDI and MAS cultural context influencing the graphical design of game interface?

Q2: Do context, IDV and MAS cultural context influencing the animation design of game interface?

\section{METHODOLOGY}


This research use triangulation method which combines quantitative and qualitative methods. Triangulation method has been found to increase confidence in the results of the analysis [9]. The first step is to distribute a set of validated questionnaire to identify player preferences based on their cultural values. Then, the feedback is analyzed and based on the analysis, the game interface is designed. Several design experts are appointed to verify the design before the game prototype is developed. Seven game players are selected to play the game prototype and are interviewed to get their feedbacks. Fig. 1 shows the method used for the research.

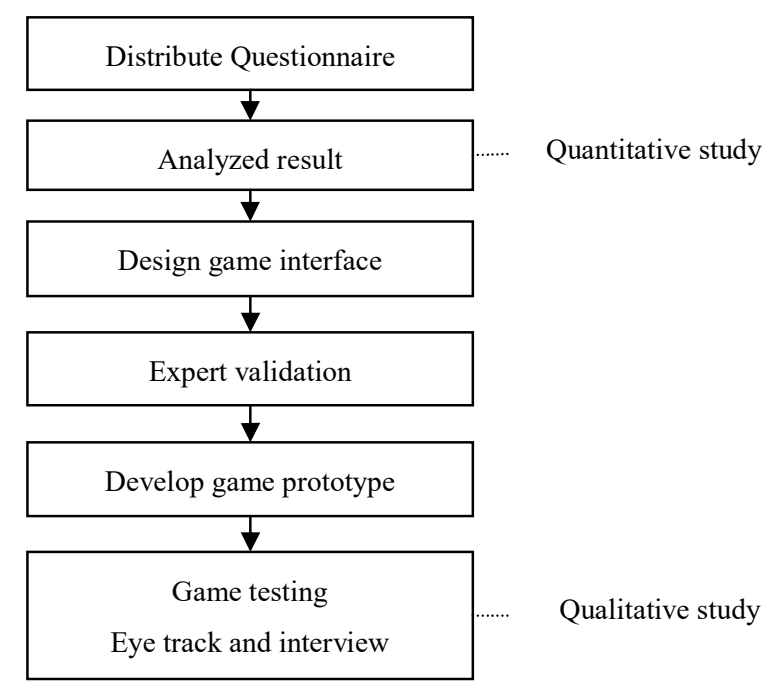

Fig.1. Research methodology

\subsection{Questionnaire}

The validated questionnaire is taken from [10]. It contained the following information:

i. Objective of the questionnaire and demographic questions.

ii. 18 questions using 10 point Likert scale.

\subsection{Subject}

265 subjects participate in the survey as shown in Table 1. The sample size is sufficient for convenient sampling and the subjects were chosen with the criterion of playing computer games more than 5 times a week.

Table 1. Units for magnetic properties 


\begin{tabular}{lll}
\hline Item & Total & \% \\
\hline Gender & & \\
Male & 150 & 57.7 \\
Female & 115 & 43.4 \\
\hline Age & & \\
$18-20$ & 107 & 40.4 \\
$21-23$ & 116 & 43.8 \\
$24-26$ & 29 & 10.9 \\
$27-30$ & 13 & 4.9 \\
\hline Race & & \\
Malay & 185 & 69.8 \\
Chinese & 62 & 23.4 \\
Indian & 18 & 6.8 \\
\hline Religion & & \\
Islam & 190 & 71.7 \\
Buddha & 23 & 8.7 \\
Hindu & 15 & 5.6 \\
\hline & & \\
\hline
\end{tabular}

\subsection{Expert Validation}

Four experts were chosen with the criterion of having experience in multimedia design more than five years. A checklist was given to the experts to validate the design of game interfaces. The checklist was adapted from the questionnaire and the objective is to identify whether all the design preferences are presented in the game interfaces.

\subsection{Testing}

Seven game players were selected to play the games. The number of respondents set based from previous studies indicates that using a total of five to ten respondents' means that $80 \%$ to $90 \%$ of problems can be traced [12]. All players consider interface design as one of the most important factors for a good game. There is only one machine that is used for the testing thus only one player can play the game at a time. The game is played on a desktop computer that is equipped with an eye tracker device. The eye tracker will track the players' eye movement and keep the record. After the player finish playing the game, he or she will be interviewed based on the eye tracker result. In a pilot study that has been done previously, a single player 
takes about one hour to finish the test.

The eye-tracking device is Tobii T60 and the application isTobii Studio ${ }^{\mathrm{TM}}$ Analysis Software which has been installed on a desktop computer with Windows XPoperating system. Eye movement signals are processed using a small camera mounted on the optical board under the monitor. Evaluation was done based on the analysis of the heat map, fixation duration and fixation count. These records are important to help the prototype evaluation [13].

The interview session starts with a set of enjoyment and presence questions which are adapted from validated questionnaires. The questionnaire has been used in previous research to evaluate game and other type of entertainment [14-15]. The players also need to describe briefly their experience in playing the game based on their cultural values.

\section{EXPERIMENTAL}

This topic is divided into several parts. The first part contains analysis of survey while the second part is game interface design based on the analysis. The third part contains expert evaluation on game interface design and the last part is the result of game prototype evaluation.

\subsection{Questionnaire Analysis}

All three cultural values that have been identified to influence the design of graphic in game interface shows positive result since the analysis of the survey shows a high mean value which is more than 7.0. The same goes to the result of animation design. Table 2 shows the mean value of each cultural value.

Table 2. Mean value of cultural value 


\begin{tabular}{ccc}
\hline Interface Element & Cultural Value & Mean Value \\
\hline Graphic & Context & 7.61 \\
& PDI & 7.14 \\
Animation & MAS & 7.25 \\
& Context & 7.74 \\
& MAS & 7.12 \\
\hline
\end{tabular}

\subsection{Game InterfaceDesign}

Donkey Sean, a strategy game is chosen to be redesign based on the analysis of survey result. It is one of the top online games that have attractive game interface design. Several additions have been made to the graphical interface of Donkey Sean such as:

a) Coconut palm

b) Bridge from coconut trees

c) Country house-MinangKabau

d) Building of KLCC

e) The local fruits-bananas, mangos teens

f) Signboards grocery s hop-Pak Mat's

g) Ramly burger stalls

h) Songkok.

There are a few animations added which are animated heart shape, animated arrow and animated grass. Fig. 2 in appendix shows some examples of original interfaces and the modified interfaces. Black rectangles indicate the changes that have been made. 


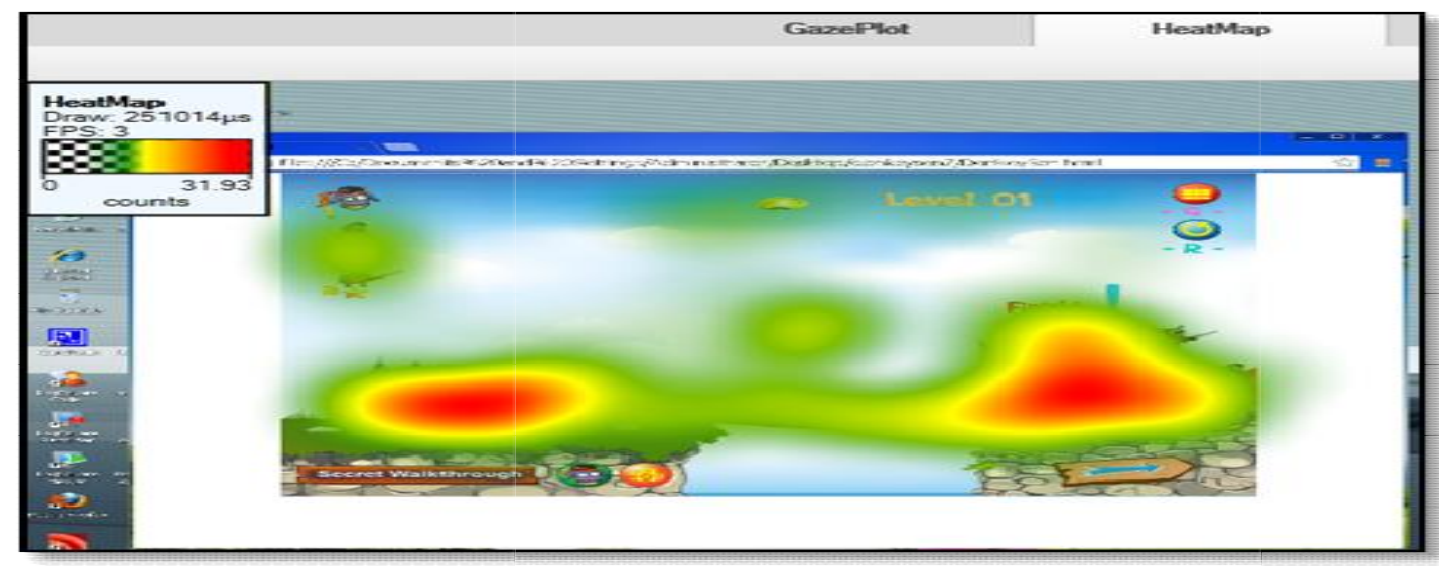

Fig.2. Example of heat map-Respondent 4

\subsection{Expert Evaluation}

Experts have been given a check list that contains eight items to evaluate the game interface design. The experts need to make sure that all eight items are presented in the modified game interfaces. Based on the evaluation result, experts agreed that all items are represented. Therefore, a game prototype is developed.

\subsection{Game Prototype Evaluation}

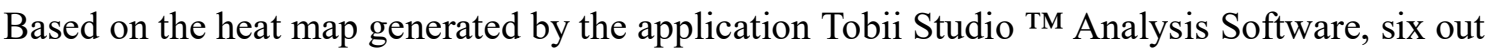
of seven respondents looked at all of the addition graphics that are mentioned earlier. Fig. 3 shows an example of heat map for respondents 4.

Original interface

Interface with cultural values

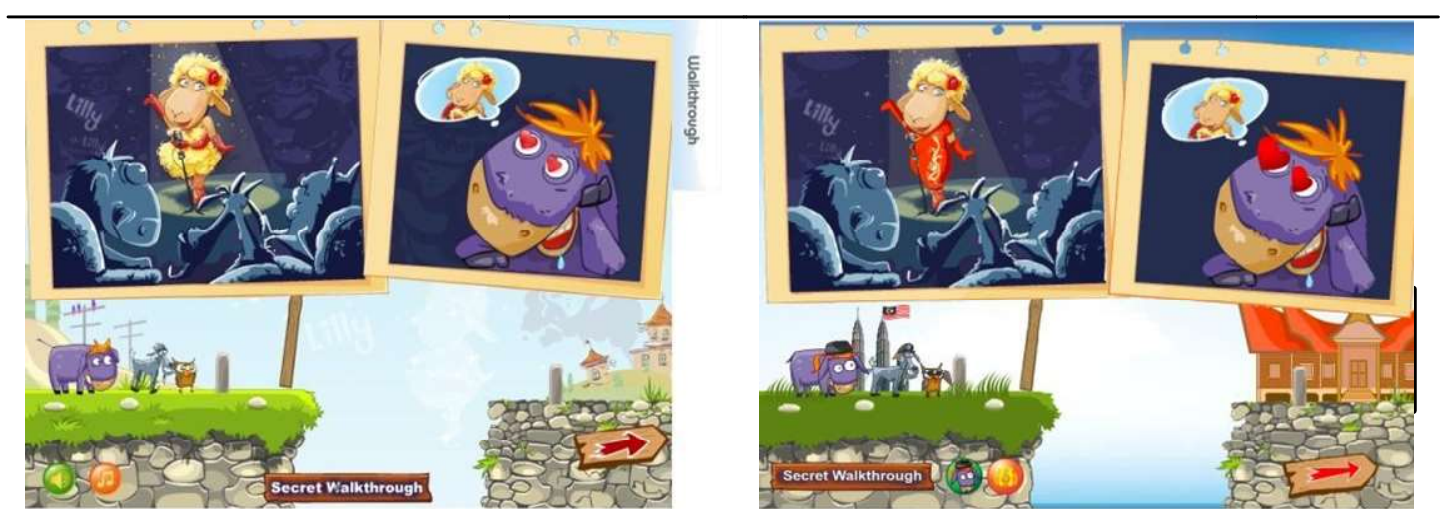




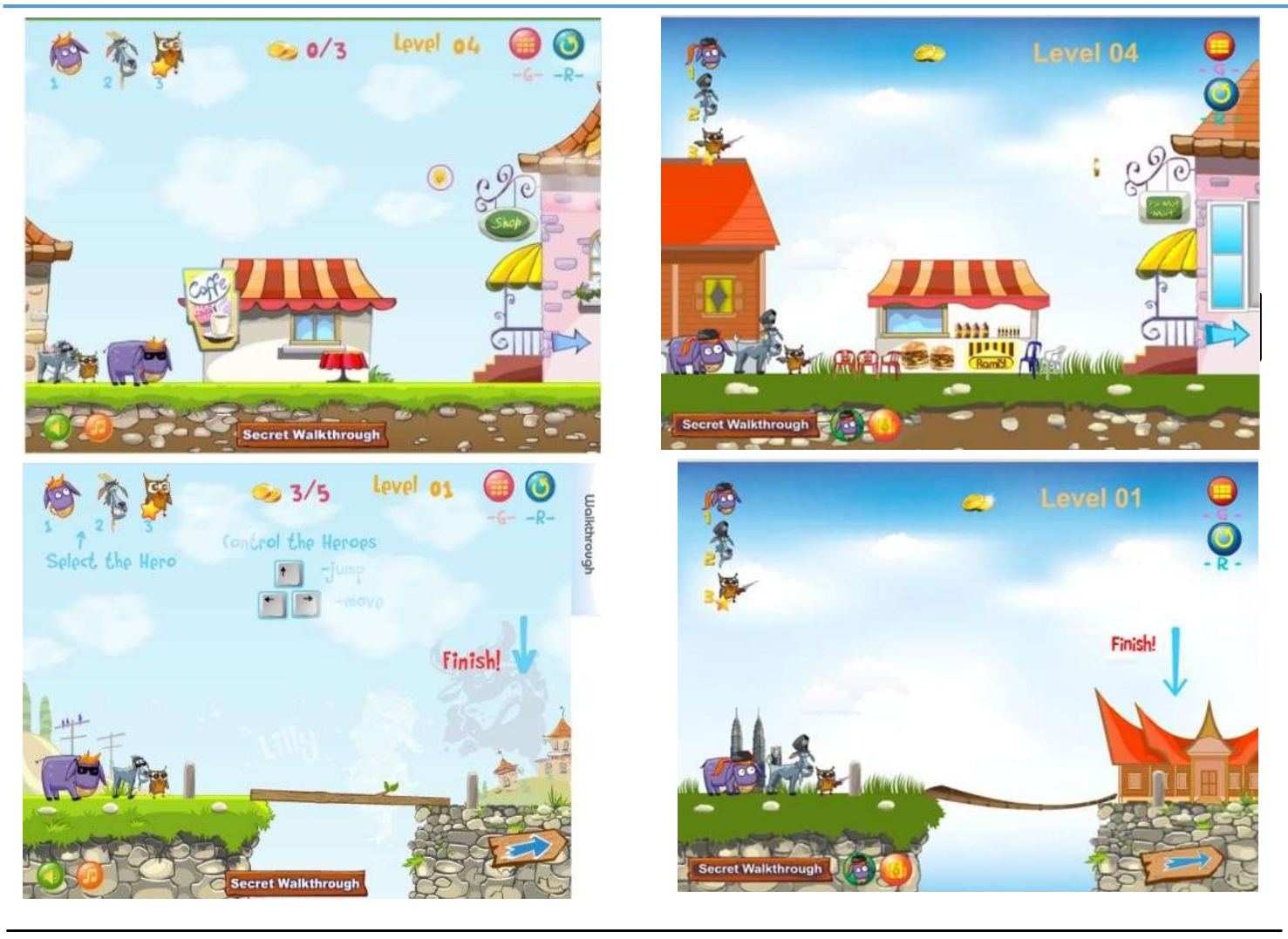

Fig.3.Examples of original interfaces and the modified interfaces

Table 3 shows the mean value of fixation duration and fixation count for the seven respondents. Based on the result of the heat map, fixation duration and count, it is identified that players give fairly enough attention to the new graphics. Analyses of interviews that have been conducted demonstrate that all players agree that Context, PDI and MAS influence the design of graphic in game interface. Table 4 shows some of the comments from the interview sessions. 
Table 3. Mean values of fixation duration and fixation count for seven respondents (milisecs)

\begin{tabular}{ccc}
\hline Graphic & Fixation Duration & Fixation Count \\
\hline Coconut & 0.25 & 4 \\
Bridge & 0.28 & 7 \\
Houses & 0.35 & 23 \\
KlCC & 0.4 & 8 \\
Fruit & 0.35 & 7 \\
Pak mat & 0.61 & 7 \\
Ramly & 0.33 & 72 \\
Songkok & 0.15 & 1 \\
Flag & 0.28 & 1 \\
\hline
\end{tabular}

Table 4.Respondents' comments on graphic

\begin{tabular}{ll} 
Respondent & \multicolumn{1}{c}{ Comments } \\
\hline 4 & I like the graphical interface than text. I can easily understand the message \\
through graphics. See new graphics that I never seen before makes me very \\
intrigued \\
The graphics are unique and pretty. Its influence my choice for playing \\
computer games. Unique and beautiful pretty graphicinfluenced my choice \\
to play computergames. If the storyline is done well, it definitely adds on. \\
I can play computer games with simple graphics. But, the graphics seem \\
more interesting and fun. Text only or little graphic may look kind of boring. \\
Combination of different graphics can make me feel like I see a game \\
design.
\end{tabular}

Six animations that are added on Donkey Sean interface design are:
a) Moving arrows
b) Birds
c) Coins
d) Heart shape
e) Owls
f) Grass 
All respondents agree with the design of fantasy animation. Six respondents gave positive responses during the interview sessions. Respondent 2 stated that he would like to be imaginative when playing and fantasy animation let him achieve his objective. Besides that, all respondents agreed that a stylish animation can add interest the game especially if other elements in the game are well-designed. Table 5 shows further comments from the selected respondents.

Table 5. Respondents' comments on animation

\begin{tabular}{cl}
\hline Respondent & \multicolumn{1}{c}{ Comments } \\
\hline 1 & I like the animation a lot. It makes the games seem alive \\
2 & Animation is the most object that I can remember after finish the play \\
4 & I can see the different types of animation added. Additional animation does not \\
& disturb me even caught my attention
\end{tabular}

The respondents have also been asked to evaluate their enjoyment and presence when playing the games and six of them identified that the game is fun and they enjoy playing the game. Thus, the study also found that the player's level of enjoyment and presence is high at $85.6 \%$.

\section{DISCUSSION AND CONCLUSION}

Results from the surveys have shown that the three cultural values, specifically context, PDI and MAS cultural context do influence the design of graphic on game interface and the same can be said about context, IDV and MAS cultural context on the animation design of game interface. The claim is further strengthen by testing the modified game interface with the identified cultural values. Responses from the selected gamers on the modified game interfaces proved that the localized design influence them in enjoying the game more and makes the playing more fun and prolonged. Adding animations on the game interface also lends excitement to the game and is non-intrusive to the game. It makes the game more memorable to the gamers.

Overall, it can be said that culture do play an important role in influencing players preferences and designer should design game interface with consideration on cultural values since it can be favorable to players enjoyment. This considerable contribution to the game players and local game industry will allow local game developers to come out with their own localized 
game interface design rather than imitate existing games in the market.

\section{FUTURE WORKS}

This study is only an initial step towards defining the relationship between cultural values and designing game interface. Most of the effort was allocated to identifying user preferences based on their cultural values. For future works on the design of game interface, other elements of interfaces such as color, menu button and layout should be addressed. Past research [16] identify these elements form part of important game interface elements that have effects on the design.

\section{ACKNOWLEDGEMENTS}

This research is funded by UniversitiKebangsaan Malaysia under the research grant, UKM-GUP-2011-236.

\section{REFERENCES}

[1] Schell J. The art of game design: A book of lenses.Florida: CRC Press, 2014

[2] Adams E.Fundamentals of game design.London: Pearson Education, 2014

[3] Hofstede G. Culture's consequences: International differences in work-related values. London: SAGE, 1984

[4] Lee Y H, Wohn D Y. Are there cultural differences in how we play? Examining cultural effects on playing social network games. Computers in Human Behavior, 2012,28(4):1307-1314

[5] Ramli R Z, Sahari N, Zin M, Azan N, Osman S. 2013. Development and validation of a questionnaire to measure game interface preferences based on cultural values. Journal of Theoretical and Applied Information Technology, 2013, 56(1):100-105

[6] Hsu CC. Comparison of gender differences in young people's blog interface preferences and designs. Displays, 2012, 33(3):119-128

[7] Waters R D, Lo K D. Exploring the impact of culture in the social media sphere: A content analysis of nonprofit organizations' use of facebook. Journal of Intercultural Communication Research, 2012, 41(3):297-319 
[8] Chakraborty J, Norcio A F.Cross cultural computer gaming. In International Conference on Internationalization, Design and Global Development, 2009, pp. 13-18

[9] Lewis M W. Iterative triangulation: A theory development process using existing case studies. Journal of Operations Management, 1998, 16(4):455-469

[10] Ramli R Z, Sahari N, Zin NA M, Othman N, Osman S. Development and validation of game interface with culture questionnaire: Graphic and animation. Procedia Technology, 2013, $11: 840-845$

[11] Krejcie R V, Morgan D W. Determining sample size for research activities. Educational and psychological measurement, 1970, 30(3):607-610

[12] Nielsen J. Usability engineering. Amsterdam: Elsevier, 1994

[13] Bhattacharyya D, Chowdhury B, Chatterjee T, Pal M, Majumdar D. Selection of character/background colour combinations for onscreen searching tasks: An eye movement. Subjective and Performance Approach. Displays, 2014, 35(3):101-109

[14] Brockmyer J H, Fox C M, Curtiss K A, McBroom E, Burkhart K M, Pidruzny J N. The development of the game engagement questionnaire: A measure of engagement in video game-playing. Journal of Experimental Social Psychology, 2009, 45(4):624-634

[15] Hsiao C C, Chiou J S. The effects of a player's network centrality on resource accessibility, game enjoyment, and continuance intention: A study on online gaming communities. Electronic Commerce Research and Applications, 2012, 11(1):75-84

[16] Yue W S, Zin N A M. usabilty evaluation for history educational games. In 2nd International Conference on Interaction Sciences: Information Technology, Culture and Human, 2012,pp. 1019-1025

\section{How to cite this article:}

Ramli R.Z, Zin N.A.M, Sahari@Ashaari N., Ismail M.N., Osman S. Design of graphic and animation in game interface based on cultural value: verification. J. Fundam. Appl. Sci., 2017, 9(5S), 183-194. 